\title{
3D Quantum-inspired Self-supervised Tensor Network for Volumetric Segmentation of Medical Images
}

\author{
Debanjan Konar, MIEEE, Siddhartha Bhattacharyya, SMIEEE, Tapan K. Gandhi, SMIEEE, \\ Bijaya K. Panigrahi, SMIEEE, and Richard Jiang, SMIEEE
}

\begin{abstract}
This paper introduces a novel shallow 3D selfsupervised tensor neural network for volumetric segmentation of medical images with merits of obviating training and supervision. The proposed network is referred to as 3D Quantuminspired Self-supervised Tensor Neural Network (3D-QNet). The underlying architecture of 3D-QNet is composed of a trinity of volumetric layers viz. input, intermediate and output layers inter-connected using an $\mathcal{S}$-connected third-order neighborhoodbased topology for voxel-wise processing of $3 \mathrm{D}$ medical image data suitable for semantic segmentation. Each of the volumetric layers contains quantum neurons designated by qubits or quantum bits. The incorporation of tensor decomposition in quantum formalism leads to faster convergence of the network operations to preclude the inherent slow convergence problems faced by the classical supervised and self-supervised networks. The segmented volumes are obtained once the network converges. The suggested 3D-QNet is tailored and tested on the BRATS 2019 Brain MR image data set and Liver Tumor Segmentation Challenge (LiTS17) data set extensively in our experiments. 3DQNet has achieved promising dice similarity as compared to the intensively supervised convolutional network-based models like 3D-UNet, Vox-ResNet, DRINet, and 3D-ESPNet, showing a potential advantage of our self-supervised shallow network on facilitating semantic segmentation.
\end{abstract}

Index Terms-Quantum Computing, Volumetric Medical Image Segmentation, QIS-Net, Tensor Network

\section{INTRODUCTION}

A utomatic volumetric medical image segmentation assisted by contextual information yields Volumes of Interest (VOIs), which are critical to cancer patients. Deeply supervised Convolutional Neural Networks (CNN) have achieved respectable accuracy in 2D medical image segmentation [1] [4]. However, in automatic 3D medical image data segmentation, deeply supervised 3D-CNNs suffer from manually affected challenges viz. acquiring sufficient $3 \mathrm{D}$ annotated data for suitable training, high heterogeneity and dimensionality of 3D medical images, complex anatomical environments and the need for optimizing the 3D neural networks [5]-[7]. Hence, 3D medical imaging research calls for self-supervised learning for accurate and fast segmentation of volumetric medical images

D. Konar, T. K. Gandhi, and B. K. Panigrahi are with the Department of Electrical Engineering, Indian Institute of Technology Delhi, New Delhi, India, Email: Debanjan.Konar@ee.iitd.ac.in, tgandhi@iitd.ac.in and bkpanigrahi@iitd.ac.in

S. Bhattacharyya is with Rajnagar Mahavidyalaya, Birbhum, West Bengal, India, Email: dr.siddhartha.bhattacharyya@gmail.com

R. Jiang is with the School of Computing and Communications, Lancaster University, Lancaster, UK, Email: r.jiang2@lancaster.ac.uk with different modalities.

The unified concept of quantum-inspired neural networks (QINN) enhances the approximation and generalization capabilities of classical neural networks and has emerged to process information faster in the field computer science [8], [9]. Of late, quantum-inspired neural networks are becoming popular in solving problems in the domain of pattern recognition and classification [10]-[13] employing the inherent characteristics of quantum computation. However, the complex and timeintensive quantum back-propagation algorithm involved in the aforementioned QINN models suffer from slow convergence problems. In addition, the fixed activation schemes adopted in the QINN models restrict their application to gray-scale image segmentation.

Given 3D medical image data, the primary aim of our proposed 3D-QNet architecture is to perform volumetric organ and lesions segmentation with expert level accuracy for tumor identification alleviating supervision or training. Our proposed 3D-QNet architecture is centered on the self-supervised bidirectional counter propagation of the quantum states obviating the time-intensive quantum back-propagation algorithm for faster convergence. The network hyper-parameters associated with the gray-level thresholding process are adaptive in nature, and voxel-wise context-sensitive information is exhibited in quantum formalism as reported in this article. The current voxel-wise segmentation work has significant contributions over 2D medical image segmentation [1]-[3], [12] as given below.

1) We propose a novel quantum-inspired self-supervised shallow voxel-wise neural network referred to as 3DQNet which has relevance on volumetric medical image segmentation.

2) In this work, an $\mathcal{S}$-connected quantum fuzzy contextsensitive voxel information is processed to integrate the appearance of low-level and high-level local image features with wide intensity variations and implicit shape of the VOIs, thereby enabling accurate volumetric segmentation of 3D medical images.

3) A novel generalized quantum-inspired self-supervised learning is proposed using a tensor representation of the weight vectors for high dimensional data which has been employed in our suggested 3D-QNet for 3D medical image segmentation. The non-tensorized implementation of 3D-QNet, referred to as 3D-QNet-NonTensor is also 
demonstrated in our experiments.

4) The convergence analysis of the proposed 3D-QNet is also demonstrated with super-linearity. The primary aim at incorporating quantum computing in our proposed 3D network architecture is to exploit the features of quantum correlation and to accelerate the speed of convergence of the network operation, simultaneously improving the discrimination ability to yield fast and accurate segmentation.

The organization of the remaining sections of the manuscript is as follows: a comprehensive literature review about various deep learning-based volumetric segmentation of medical images and the challenges are presented in Section II. Section III illustrates the fundamental concepts of quantum computing. The novel self-supervised 3D-QNet architecture with quantum-inspired tensor network model is introduced in Section IV. Section V elucidates voxel-wise segmentation of $3 \mathrm{D}$ medical images using the proposed 3D-QNet. The experimental outcome and discussions are provided in Section VI. Section VII states the concluding remarks of the proposed work and sheds light into the future directions of research.

\section{RELATED WORKS}

Recent years have witnessed a surge in the application of deep learning networks in various tumor segmentation [14][18] and achieved respectable accuracy in 2D medical image segmentation [2], [19]. However, in contrast to automated volumetric segmentation of medical images, 2D convolutional neural network architectures (CNNs) [14], [16], [17] process the medical images in a $2 \mathrm{D}$ independent slice-wise fashion which leads to non-optimal use of 3D contextual feature information of volumetric medical image data (3D Computed Tomography (CT) and Magnetic Resonance Imaging (MRI)) . In turn, 3D CNN based architectures extract rich spatial and contextual features and perform voxel-wise segmentation of volumetric medical images [20]-[22]. Kamnitas et al. [20] suggested a dual path 3D CNN incorporating local and larger contextual feature information to obviate the computationally complex 3D medical image processing and to exhibit dense inference on medical image segmentation. A flexible network, 3D-UNet architecture [22] achieved remarkable success on brain MR image semantic segmentation. Of late, to exploit the 3D contextual information, Brebisson et al. [23] employed 2D CNNs on three orthogonal 2D patches and formed 3D patches in combination to reduce the memory requirements. However, 3D CNN networks suffer from slow convergence problems owing to computationally exhaustive 3D convolution operations and extensive training procedures. Despite popularity among the medical and computer vision researchers, UNet architectures [22] fall short in scalability and are unable to distinguish the distinctive features (shape, size, intensity, location etc.) learned at the convolutional layers. Moreover, it suffers from the vanishing gradient problem when the number of feature layers is increased for better representation of the features. Various deeper network architectures obviating the vanishing gradient problem have been proposed concurrently for voxel-wise medical image segmentation including VoxResNet [24], DRINet [25] and 3D-ESPNet [26]. However, these deeply supervised network architectures suffer in computational complexity and slow-convergence with an increase in the number of feature layers in the network architecture. Currently, self-supervised/semi-supervised/weakly supervised networks have gained significant attention among computer vision and medical research community due to lack of annotated images for deep supervision [27]-[30]. Nevertheless, these self-supervised networks [27]-[30] for volumetric medical image segmentation rely on pre-trained 3D CNN models, and hence these are not fully self-supervised networks. Moreover, these networks are characterized by significant memory footprints and often this is a serious obstacle in employing in various medical imaging application settings. It inspires us to develop 3D self-supervised neural network architectures for volumetric medical image segmentation.

The main problem with the classical self-supervised neural network models lies in the fact that they do not converge fast and hence the segmented outcome is distorted due to the slower convergence problems [31], [32]. Numerous quantum neural networks have been evolved in the last few decades replicating classical neural networks and offering faster processing while compared with the classical counterparts [9], [33]-[36]. The quantum versions of the classical self-supervised neural network architectures [10], [11], [37], [38] offer a potential candidate for faster and efficient image segmentation and surpasses the classical counterparts. Konar et al. recently developed quantum-inspired neural network models referred to as QIS-Net [12], QFS-Net [13] and QIBDSNet [39] suitable for brain MR image segmentation. These networks have been found to attain promising outcome in complete brain tumor segmentation and serves the motivation behind assimilation of quantum-inspired computing in the current 3D-QNet architecture.

In this manuscript, a novel fully self-supervised quantuminspired shallow neural network architecture is presented for volumetric medical image segmentation to obviate the comprehensive challenges faced by deep supervision of complex 3D CNNs.

\section{Fundamentals OF QuANTUM COMPUTING}

The basic concept of quantum computing deals with the principles of quantum mechanics and offers to demonstrate the quantum computing algorithms which rely on quantum bits having quantum operations on qubits [40].

\section{A. Quantum Bits and Tensor Products}

The basic element equivalent to classical bits in quantum computing is known as quantum bit or qubit and is represented using Dirac notations $|0\rangle$ and $|1\rangle$. However, unlike classical computing, quantum bits are expressed as a linear combination of probability amplitudes often known as superposition as follows [9].

$$
|\phi\rangle=\cos \frac{\alpha}{2}|0\rangle+e^{i \frac{\theta}{2}} \sin \frac{\alpha}{2}|1\rangle
$$

where, $0 \leq \alpha \leq \pi$ and $0 \leq \theta \leq 2 \pi$.

Hence, qubits reside in the Hilbert space parametrized by the continuous variables $\theta$ and $\alpha$. In quantum formalism, the 
tensor products of the subspace form the full Hilbert space, $H$ as

$$
H=\otimes_{t=1}^{n} H_{t}
$$

A set of $n$ basis states (designated as $\left|\phi_{j}\right\rangle$ ) comprising $0-1$ can form a qubit system $|\psi\rangle$, of size $\log n$ in the Hilbert space, $H$ as follows.

$$
|\psi\rangle=\sum_{j}^{n} p_{j}\left|\phi_{j}\right\rangle
$$

where, $p_{j}$ is the probability amplitude and $\left|\phi_{j}\right\rangle=\left|\phi_{1}\right\rangle \otimes$ $\left|\phi_{2}\right\rangle \otimes \ldots\left|\phi_{n}\right\rangle$. For example, using two qubits, four distinct tensor sub-spaces can be created as basis $|0\rangle \otimes|0\rangle,|0\rangle \otimes|1\rangle$, $|1\rangle \otimes|0\rangle$ and $|1\rangle \otimes|1\rangle$ often represented as $|00\rangle,|01\rangle,|10\rangle$, and $|11\rangle$, respectively.

\section{B. Input Data Encoding and Tensor Decomposition}

A tensor product basis relies on the local input feature map $\left\{\boldsymbol{\Phi}^{d_{j}}\left(\alpha_{j}\right)\right\}$ in the Hilbert space of functions over $\alpha_{j} \in[0,1]$ as [36]

$\left|\Phi^{d_{1}, d_{2}, \ldots d_{N}}(\boldsymbol{\alpha})\right\rangle=\left|\phi^{d_{1}}\left(\alpha_{1}\right)\right\rangle \otimes\left|\phi^{d_{2}}\left(\alpha_{2}\right)\right\rangle \otimes \ldots \otimes\left|\phi^{d_{N}}\left(\alpha_{N}\right)\right\rangle$

where, $d_{j}$ varies from $1 \ldots N$ ( $N$-dimensional vector). A function, $f^{l}(\boldsymbol{\alpha})$ can be realized using the tensor product of input local feature map $\phi^{d_{j}}\left(\alpha_{j}\right)$ and the network weight decomposition $\boldsymbol{\Psi}$, as follows.

$$
\begin{array}{r}
f^{l}(\boldsymbol{\alpha})=\Psi^{l} \cdot \boldsymbol{\Phi}(\boldsymbol{\alpha}) \\
=\left|\boldsymbol{\Psi}_{d_{1} d_{2} \ldots d_{N}}\right\rangle\left|\phi\left(\alpha_{1}^{d_{1}}\right)\right\rangle \otimes\left|\phi\left(\alpha_{2}^{d_{2}}\right)\right\rangle \ldots \otimes\left|\phi\left(\alpha_{N}^{d_{N}}\right)\right\rangle
\end{array}
$$

Hence, the local feature map $\phi^{d_{j}}\left(\alpha_{j}\right)$ forms a basis for a Hilbert space of functions defined over $\alpha \in[0,1]$ and the tensor product basis $\boldsymbol{\Phi}^{d_{1}, d_{2}, \ldots d_{N}}(\boldsymbol{\alpha})$ forms a Hilbert space of functions defined over $\alpha \in[0,1]^{N}$. Considering the dimensions of the input feature vector restricted to $N=2, \phi(\alpha)$ is defined as

$$
\phi(0)=[0,1], \phi(1)=[1,0]
$$

In order to enhance and extract the contextual information from high dimensional data, Trcuker tensor decomposition is suitable for neural network layer decomposition [41]. The inner product of the two equal size tensors $\mathbf{V}, \Psi \in \mathbb{R}^{m \times n \times p}$ defined as follows.

$$
\begin{array}{r}
|\boldsymbol{\xi}\rangle=\mathbf{V} * \boldsymbol{\Psi}=\sum_{l}\left\langle\mathbf{V}^{(l)}, \boldsymbol{\Psi}^{(l)}\right\rangle=\sum_{i=1}^{M} \sum_{j=1}^{N} \phi_{i, j} \theta_{i, j}, \\
\phi_{i, j} \in \mathbf{V}, \theta_{i, j} \in \boldsymbol{\Psi}
\end{array}
$$

\section{3D QUANTUM-INSPIRED SELF-SUPERVISED TENSOR Neural Network (3D-QNet) ARChitecture}

In this article, a 3D Quantum-inspired Self-supervised Tensor Network (3D-QNet) with self-supervised tensor learning is proposed for automatic voxel-wise segmentation of medical images. The 3D-QNet comprises trinity of volumetric layers of quantum neurons arranged as input, intermediate and output layers. A schematic outline of the proposed 3DQNet architecture is shown in Figure 1. The input volume
$(M \times N \times P)$ is normalized and propagated from the 3D input layer to the successive 3D hidden and output layers of the 3DQNet architecture for processing through $\mathcal{S}$-connected voxels. Each of the three volumetric layers of 3D-QNet architecture is fully intra-linked with qubits using a 3D-matrix representation. Each 3D layer of the proposed architecture is intra-connected through quantum neurons with intra-connection strengths set to $\frac{\pi}{2}$ (quantum 1 logic). The basic processing unit of each volumetric layer of the 3D-QNet architecture is an $\mathcal{S}$-connected neighborhood-based voxel-wise orientation of each candidate neuron as illustrated in Figure 1. The inter-layer connection between the 3D input to 3D intermediate, 3D intermediate to 3D output layer is formed using the $\mathcal{S}$-connected voxel-wise neighborhood orientation. The contribution of the $\mathcal{S}$ number of neighborhood quantum neurons (pixels) of a candidate neuron at one 3D layer is propagated in the forward direction and accumulated at the corresponding candidate neuron of the subsequent 3D layer. Consequently, the voxel-wise information from the 3D output layer to 3D intermediate layer is counter-propagated for further processing. The voxel-wise processing of each 3D layer is performed along with the depth of the 3D layer for semantic segmentation. The inter-linked connections between two successive 3D layers are represented using 3D weight matrices of qubits and each inter-connection weights are updated using rotation gate for faster processing. The relevant details about the principle of operation of the proposed 3D-QNet for volumetric segmentation are provided in the following subsections using a self-supervised Tensor learning model in quantum formalism.

\section{A. 3D-Quantum-Inspired Self-supervised Tensor Network Model}

In the suggested 3D-QNet architecture, the high dimensional weight vector $\Psi$ is represented using tensor to optimize the network operations and to facilitate the extraction of significant semantic feature information in the quantum-inspired self supervised model. The internal kernels associated with the network operate in parallel, thereby accelerating convergence of the 3D-QNet. The input quantum neurons containing the pixel intensity are expressed as qubits and the inter-connection weights are represented using quantum rotation gates. The classical intensity of any $i^{t h}$ normalized gray-scale image pixel of MR or CT volume (denoted as $\alpha_{i} \in[0,1]$ ) is transformed into quantum state using a mapping function $\phi\left(\alpha_{i}\right)$ as follows.

$$
\phi\left(\alpha_{i}\right)=\left[\cos \left(\frac{\pi}{2} \alpha_{i}\right) \sin \left(\frac{\pi}{2} \alpha_{i}\right)\right] \forall i=1, \ldots M, j=1, \ldots N
$$

The angle of rotation is measured using the relative difference of fuzzy intensity of the candidate pixel and the neighborhood pixels in quantum formalism. This relative measure helps to segment the foreground and background regions of an image.

$$
\omega_{i, j}=1-\left(\alpha_{i}-\alpha_{i, j}\right) ; j \in\{1,2,3, \ldots \mathcal{S}\}
$$

Hence, $\omega_{i, j}$ is designated as the angle of rotation and measured as the relative intensity difference between the candidate pixel $\left(\alpha_{i}\right)$ and one of its neighborhood pixels $\alpha_{i, j}$. The strength of inter-connection between neuron $j$ (neighborhood of the 


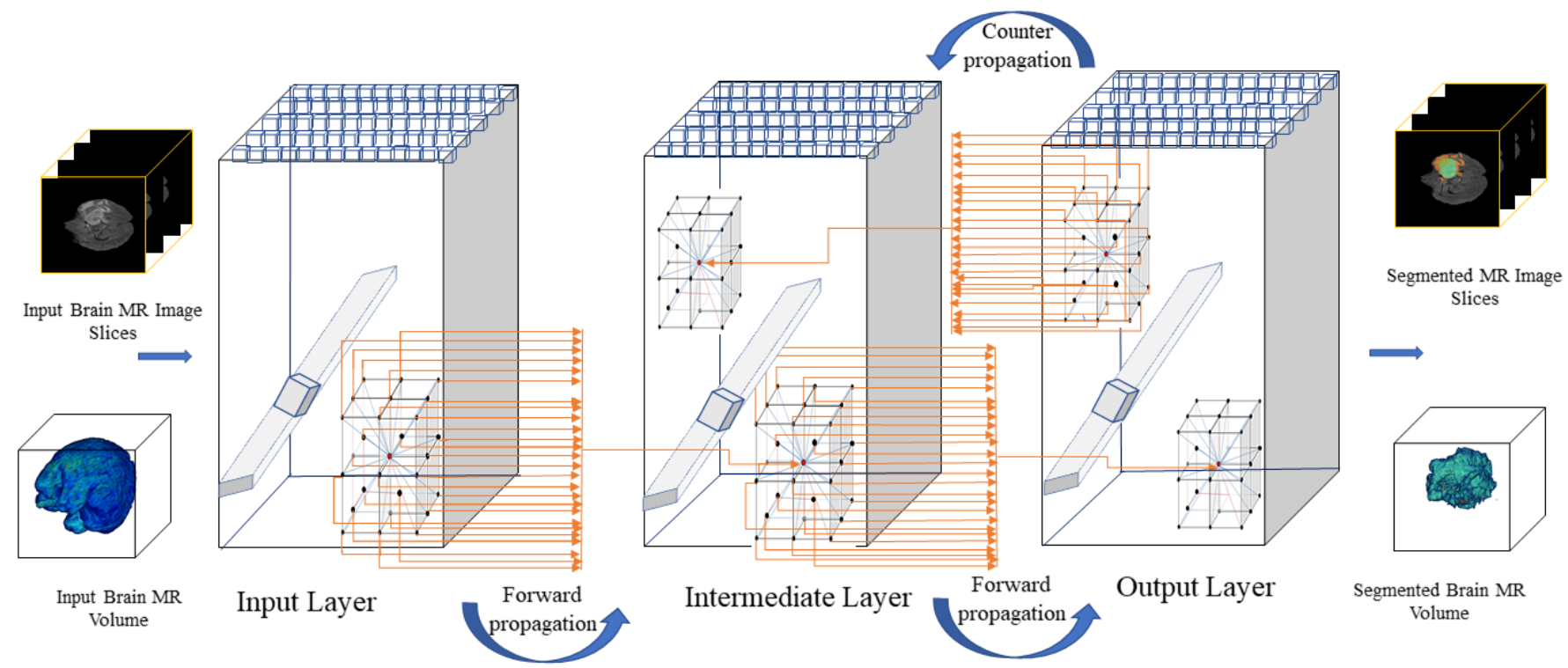

Fig. 1: 3D Quantum-inspired Self-supervised Tensor Neural Network (3D-QNet) architecture (Only three Inter-layer connection is illustrated for better visibility).

candidate neuron $i$ ) of a layer to the corresponding candidate neuron of the adjacent layer is mapped using $\varphi$. The classical interconnection weight $[0,1]$ is transformed into quantum formalism as

$$
\left|\varphi\left(\omega_{i}\right)\right\rangle=\left[\cos \left(\frac{\pi}{2} \omega_{i}\right) \sin \left(\frac{\pi}{2} \omega_{i}\right)\right]
$$

In this proposed tensor network model, the 3D-QNet layer is decomposed as voxel (core tensor) using Tucker Tensor decomposition [41] to reduce the input dimensions and the interconnection wight as factor matrices. Let us consider tensor $\mathcal{V}, \Psi \in \mathbb{R}^{m \times n \times p}$, where $\mathcal{V}$ is the voxel-wise input of $3 \mathrm{D}$ MR images and the corresponding inter-connection 3D weight matrix as evaluated in Eq. 10, respectively ( $m, n, p$ denote the row, column and slice number. $\mathcal{V}, \Psi$ are third order tensors $(1 \leq m \leq M, 1 \leq n \leq N, 1 \leq p \leq P))$. According to Tucker Tensor decomposition [41],

$$
\mathcal{X}=\mathcal{V} \times_{1} \boldsymbol{\Psi}^{1} \times_{2} \boldsymbol{\Psi}^{2} \times_{3} \boldsymbol{\Psi}^{3}
$$

where, $\mathcal{X} \in \mathbb{R}^{m \times n \times p}$ the tensor outcome, $\Psi^{n}$ is the weight matrix in terms of $n$ factor matrix and $\times_{n}$ is the $\bmod -n$ product of tensor with a matrix. Each layer of the proposed 3DQNet architecture is transformed to lower dimensional tensors. Such types of $M \times N \times P$ tensors in terms of voxel are formed for each layer in the underlying network architecture. Each volumetric layer of the 3D-QNet architecture forms $M \times N \times P$ volumetric patches (voxels) of size $\mathcal{S}$ corresponding to the candidate pixels as

$$
\boldsymbol{v}=\operatorname{vox}(\mathcal{V})
$$

Here, $\mathcal{V}$ comprises all 3D-patches (voxels), $\boldsymbol{v} \in \mathbb{R}^{m \times n \times p}$ for a network layer in the proposed $3 \mathrm{D}-\mathrm{QNet}$ architecture. The spatial features in terms of neighborhood pixels of every seed pixel at the network layer are extracted and propagated to the next subsequent layers as inputs guided by a Quantum-inspired voxel-wise multi-level Sigmoidal (Vox-QSig) activation function, $\sigma_{3 D-Q N e t}$ as follows.

$$
\boldsymbol{y}^{l}=\sigma_{3 D-Q N e t}\left(\boldsymbol{v}^{l-1} * \varphi^{l}(\omega)\right)
$$

where, $\boldsymbol{v}^{l-1} \in \mathbb{R}^{M \times N \times P}, \varphi^{l}(\omega) \in \mathbb{R}^{M \times N \times P \times K}$ at the network layer $l=2,3, \boldsymbol{y}^{l} \in \mathbb{R}^{K}$ and $*$ is the inner product operator. The fuzzy context-sensitive activation (designated as $\chi_{i}$ ) for semantic segmentation in quantum formalism is defined as follows.

$$
\left|\chi_{i}\right\rangle=\left[\begin{array}{c}
\cos \vartheta_{i} \\
\sin \vartheta_{i}
\end{array}\right]
$$

where, the angle of rotation, $\vartheta_{i}$ is evaluated using the summation of the intensities of third order $\mathcal{S}$-connected neighborhood pixels (denoted as $\alpha_{i, j}, j=1,2, \ldots K$ ) of a candidate pixel $i$ (neuron) in quantum formalism using the following equation.

$$
\vartheta_{i}=2 \pi \times\left(\sum_{j=1}^{K} \alpha_{i, j}\right)
$$

Quantum fuzzy context-sensitive thresholding determines the bi-directional propagation of quantum information between the layers of the 3D-QNet architecture by means of selforganization of the inter-linked weight matrices. Reduction of feature dimensions using tensor decomposition followed by voxel-wise information processing of the proposed 3D-QNet architecture is inspired by the basic quantum neural network input-output model [12] as follows:

$$
\left|\phi^{l}\left(\alpha_{i}^{d}\right)\right\rangle=\sigma_{3 D-Q N e t}\left(\sum_{j}^{m \times n \times p} f^{l-1}\left(\alpha_{i}^{d}\right)\left\langle\varphi_{j}^{l} \mid \chi_{i}^{l, d}\right\rangle\right)
$$

where, $\left|\phi^{l}\left(\alpha_{i}^{d}\right)\right\rangle$ denotes the intermediate output of the $i^{t h}$ seed quantum neuron at the 3D network layer in the $l^{\text {th }}$ sample with depth (slice\#) $d=1,2, \ldots P . \sigma_{3 D-Q N e t}$ is the Quantum-inspired voxel-wise multi-level Sigmoidal activation (Vox-QSig) function with activation as $\left|\chi_{i}^{l, d}\right\rangle$ described in the 
following subsection IV-B. The output $\left|\phi^{l}\left(\alpha_{i}^{d}\right)\right\rangle$ can be written as

$$
\begin{array}{r}
\left|\phi^{l}\left(\alpha_{i}^{d}\right)\right\rangle= \\
f\left(\frac{\pi}{2} \delta_{i}^{l, d}-\arg \left\{\sum_{j}^{m \times n \times p} f^{l}\left(\omega_{j, i}^{d}\right) f^{l-1}\left(\alpha_{i}^{d}\right)-f^{l}\left(\chi_{i}^{d}\right)\right\}\right) \\
=\sigma_{3 D-Q N e t}\left(\sum _ { j } ^ { m \times n \times p } f ^ { l - 1 } ( \alpha _ { i } ^ { d } ) \left(\cos \left(\omega_{j, i}^{l, d}-\vartheta_{i}^{l}\right)+\right.\right. \\
\left.\left.\gamma \sin \left(\omega_{j, i}^{l, d}-\vartheta_{i}^{l}\right)\right)\right)
\end{array}
$$

Here, the designated rotation angles associated with the interconnection weights between input neuron $j$ to output neuron $i$ are represented by $\omega_{j, i}^{l, d}$ and $\delta_{i}^{l, d}$ is the phase transfer parameter. The true classical output state $(|1\rangle)$ from the $i^{\text {th }}$ quantum neuron is obtained considering the imaginary part $(\sin )$ of the above expression where, $\gamma$ is an imaginary unit. Assume that the inter-connection weights between the input and hidden layer of the 3D-QNet architecture are denoted by $\left|\Psi_{k, j}^{l, d}\right\rangle$ and for the hidden layer to output layer are denoted by $\left|\Psi_{j, i}^{l, d}\right\rangle$ in the $l^{t h}$ sample sets. The activation at the hidden and output layers are designated using $\left|\chi_{j}^{l, d}\right\rangle$ and $\left|\chi_{i}^{l, d}\right\rangle$, respectively. Considering any quantum seed neuron $k$ from the sample of input neurons at the input layer, the corresponding seed neuron at the hidden layer be $j$ and the output seed neuron be $i$, the response at the $i^{t h}$ neuron with depth $d$ in the $l^{t h}$ sample sets is expressed as

$$
\begin{array}{r}
\left|\phi^{l}\left(\alpha_{i}^{d}\right)\right\rangle=\sigma_{3 D-Q N e t}\left(\sum_{j}^{m \times n \times p} f\left(\frac{\pi}{2} y_{j}^{l, d}\right)\left\langle\varphi_{j i}^{l, d} \mid \vartheta_{j}^{l, d}\right\rangle\right) \\
=\sigma_{3 D-Q N e t}\left(\sum _ { j } ^ { m \times n \times p } f \left(\frac{\pi}{2} \times \sigma_{3 D-Q N e t}\left(\sum_{k}^{m \times n \times p} f\left(\frac{\pi}{2} y_{j}^{l, d}\right)\right.\right.\right. \\
\left.\left.\left\langle\varphi_{k j}^{l, d} \mid \vartheta_{k}^{l, d}\right\rangle\right)\left\langle\varphi_{j i}^{l, d} \mid \vartheta_{j}^{l, d}\right\rangle\right)
\end{array}
$$

i.e.,

$$
\begin{array}{r}
\left|\phi^{l}\left(\alpha_{i}^{d}\right)\right\rangle=\sigma_{3 D-Q N e t}\left(\sum_{j}^{m \times n \times p}\right. \\
f\left(\frac{\pi}{2} \times \sigma_{3 D-Q N e t}\left(\sum_{k}^{m \times n \times p} f\left(\frac{\pi}{2} y_{j}^{l, d}\right) \cos \left(\omega_{k, j}^{l, d}-\vartheta_{j}^{l, d}\right)\right.\right. \\
\left.\left.\left.\cos \left(\omega_{j, i}^{l, d}-\vartheta_{i}^{l, d}\right)+\gamma \sin \left(\omega_{k, j}^{l, d}-\vartheta_{j}^{l, d}\right) \sin \left(\omega_{j, i}^{l, d}-\vartheta_{i}^{l, d}\right)\right)\right)\right)
\end{array}
$$

where, $\gamma$ is an imaginary number.

\section{B. Quantum-inspired Voxel-wise multi-level Sigmoidal (Vox-} QSig) activation function

In this 3D-QNet architecture, a Quantum-inspired voxelwise multi-level Sigmoidal (Vox-QSig) activation function for voxel-wise processing of $\mathcal{S}$-connected spatially oriented neighborhood-based pixels. The Vox-QSig activation function, $\sigma_{3 D-Q N e t}$ with slope $\lambda$ and activation $v$, is defined as

$$
\sigma_{3 D-Q N e t}(x)=\frac{1}{\beta_{\tau}+e^{-\lambda(x-v)}}, 0 \leq \beta_{\tau} \leq \frac{\pi}{2}
$$

where, $\beta_{\tau}$ describes the multi-level class responses exhibited by the $\mathcal{S}$-connected third order neighborhood pixels expressed as

$$
\beta_{\tau}=\frac{\chi_{N}}{\rho_{\tau}-\rho_{\tau-1}}
$$

where, $\rho_{\tau}$ and $\rho_{\tau}$ are the $\tau^{t h}$ and $(\tau-1)^{t h}$ class outcomes, respectively and the contribution of the $\mathcal{S}$-connected neighborhood gray-level pixels is $\chi_{N}$. The generalized form of the Vox-QSig activation function is obtained by leveraging the activation function hyper-parameters employed in Equation 20 as

$$
\sigma_{V o x-Q S i g}\left(x ; \beta_{\tau}, \rho_{\tau}\right)=\sum_{\tau=1}^{L} \frac{1}{\beta_{\tau}+e^{-\lambda\left(x-(\tau-1) \rho_{\tau-1}-v\right)}}
$$

where, $L$ corresponds to the number of class levels. The multiclass responses for various hyper-parameters employed in the Vox-QSig activation functions are provided in Figure 2.

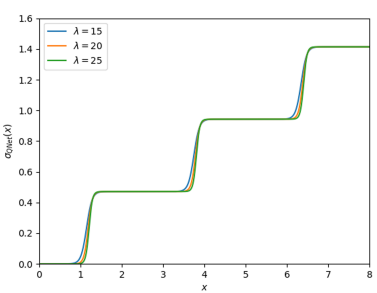

(a) $L=3$

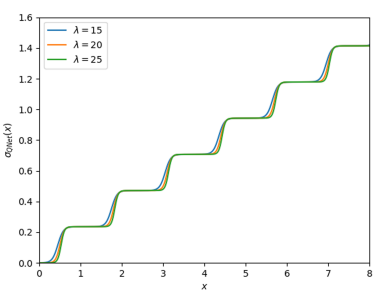

(c) $L=6$

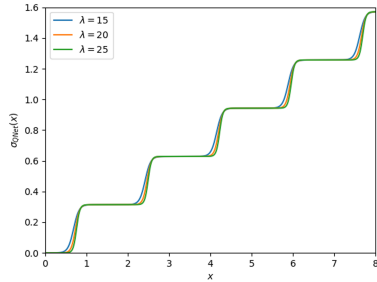

(b) $L=5$

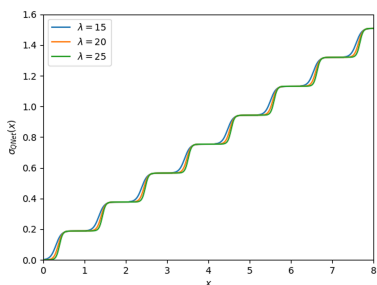

(d) $L=8$
Fig. 2: Multi-level class response of Vox-QSig activation function for $\lambda=15,20,25$.

\section{Adjustment of Inter-connection Weights of 3D-QNet and Loss Function}

Each inter-connection link for each candidate pixel of the $\mathcal{S}$-connected medical image volume and its corresponding activation are updated using quantum rotation gates thereby enabling faster convergence of the proposed 3D-QNet architecture. The inter-connection weight, $\varphi^{l, d}$ and its activation, $\chi^{l, d}$ are updated as follows.

$$
\begin{aligned}
\left|\varphi^{l+1, d}\right\rangle & =\left(\begin{array}{cc}
\cos \triangle \omega^{l+1, d} & -\sin \triangle \omega^{l+1, d} \\
\sin \triangle \omega^{l+1, d} & \cos \triangle \omega^{l+1, d}
\end{array}\right)\left|\varphi^{l, d}\right\rangle \\
\left|\chi^{l+1, d}\right\rangle & =\left(\begin{array}{cc}
\cos \triangle \vartheta^{l+1, d} & -\sin \triangle \vartheta^{l+1, d} \\
\sin \triangle \vartheta^{l+1, d} & \cos \triangle \vartheta^{l+1, d}
\end{array}\right)\left|\chi^{l, d}\right\rangle
\end{aligned}
$$

where,

$$
\omega^{l+1, d}=\omega^{l, d}+\triangle \omega^{l, d}
$$


and

$$
\vartheta^{l+1, d}=\vartheta^{l, d}+\triangle \vartheta^{l, d}
$$

Equations 25 and 26 refer to updating the angles of rotation and activation, respectively. The error or loss function, $\zeta\left(\omega^{l, d}, \vartheta^{l, d}\right)$ in the suggested 3D-QNet is evaluated in terms of Root Mean Square Error (RMSE) of the 3D-weight matrices at depth $d$ (or the slice \#d) in the $l^{t h}$ epoch and is defined on the phase angles $\omega^{l, d}, \vartheta^{l, d}$ as

$$
\begin{array}{r}
\zeta\left(\omega^{l, d}, \vartheta^{l, d}\right)= \\
\frac{1}{\mathcal{N}} \sum_{i=1}^{\mathcal{N}} \sum_{j=1}^{\mathcal{S}}\left[\varphi_{i j}\left(\omega_{i j}^{l+1, d}, \vartheta_{i}^{l+1, d}\right)-\varphi_{i j}\left(\omega_{i j}^{l, d}, \vartheta_{i}^{l, d}\right)\right]^{2}
\end{array}
$$

The convergence analysis of the proposed 3D-QNet is experimentally demonstrated with non-tensorized implementation of the network (3D-QNet-NonTensor) as shown in Figure 3. It is

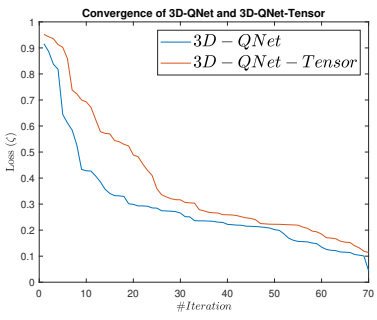

(a) $v_{\beta}$

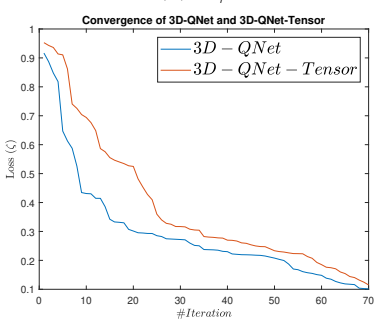

(c) $v_{\xi}$

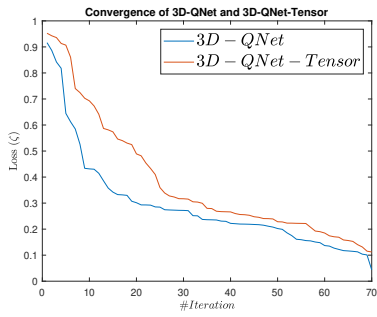

(b) $v_{x}$

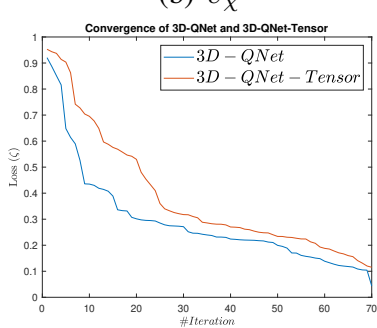

(d) $v_{\kappa}$
Fig. 3: Convergence comparison between 3D-QNet and 3DQNet-NonTensor for four distinct activation schemes

quite evident from Figure 3 that the non-tensorized implementation (3D-QNet without tensor decomposition) underperforms in terms of average convergence than 3D-QNet. It serves inspiration behind incorporation of tensor-decomposition in 3D-QNet implementation.

\section{Volumetric Medical Image Segmentation using 3D-QNET}

Minimum pre-processing is performed before the medical image slices are fed as inputs to 3D-QNet. The input medical image volume is normalized as fuzziness measure $([0,1])$ before transforming it to qubits as follows.

$$
\alpha_{i, j}^{d}=\frac{\alpha_{i, j}^{d}-\min \left(\alpha_{i, j}^{d}\right)}{\max \left(\alpha_{i, j}^{d}\right)-\min \left(\alpha_{i, j}^{d}\right)}
$$

Medical image volumes exhibit heterogeneous responses over the local intensities in the $\mathcal{S}$-connected neighborhood regions, owing to the wide variations of gray-levels. Inspired by the authors' previous works [12], [32], [42], the proposed Vox-QSig activation function employs four different adaptive thresholding schemes suitable for efficient gray-scale segmentation in the 3D-QNet architecture.

(1) Activation guided by $\beta$-distribution of the intensity of $\mathcal{S}$ connected neighborhood voxels $\left(v_{\beta}\right)$.

(2) Activation guided by $\mathcal{S}$-connected voxels based on Skewness $\left(v_{\chi}\right)$.

(3) Activation guided by $\mathcal{S}$-connected heterogeneous voxel intensities $\left(v_{\xi}\right)$.

(4) Activation guided by $\mathcal{S}$-connected fuzzy voxel cardinality estimates $\left(v_{\kappa}\right)$.

In addition, to investigate a number of optimal thresholds $\left\{\mathcal{T}_{1}, \mathcal{T}_{2}, \cdots, \mathcal{T}_{C_{l}-1}\right\}$ in multi-class settings, Otsu's method [43] is explored. The optimal thresholds maximize the class variance as follows [43].

$$
\mathcal{O}=h_{n}\left\{\mathcal{T}_{1}, \mathcal{T}_{2}, \cdots, \mathcal{T}_{C_{l}-1}\right\} \theta_{i}\left(\mu_{i}-\omega\right)
$$

where $C_{l}$ represents the number of defined classes in $\mathcal{C}$ $=\left\{C_{1}, C_{2}, \ldots, C_{C_{l}}\right\}$ and

$$
\theta_{i}=\sum_{i \in C_{l}} p_{i}, \mu_{i}=\sum_{i \in C_{l}} i p_{i} / \theta_{l}
$$

where, the $i^{\text {th }}$ pixel is defined as $p_{i}$. The probability of class $C_{i}$ is represented as $\mu_{i}$ and its mean value is given by $\mu_{i} . \omega$ is known to be the mean of class $\mathcal{C}$.

To refine the segmentation accuracy and dice score for false-positive reduction in brain tumor detection, 3D-QNet segmented highly representative volumetric intensity features are post processed using k-Means algorithm [44] for initial label segmentation and segmented into pre-defined number of clusters.

\section{RESULTS AND DISCUSSION}

\section{A. Data Set}

The proposed 3D-QNet and its non-tensorized implementation (3D-QNet-NonTensor) are validated extensively using the BRATS 2019 data set [45] and the Liver Tumor Segmentation Benchmark (LiTS17) data set [46]. The BRATS 2019 data set is composed of 315 (239 HGG and 76 LGG) 3D MRI volumes. Each MRI volume comprises 155 slices of resolution $240 \times 240$ with the ground truth segmented labels and includes four different modalities of 3D MR images viz. $T 1, T 1$ with Contrast-Enhanced (T1-CE),T2 and FLAIR. The segmented labels are annotated with three distinct tumour subregions, viz. tumor core (TC), tumor enhancing (TE), necrosis and non-enhancing core region. These three annotations form a complete tumor (WT). The BRATS 2019 data set is divided into $8: 2$ ratio for training (252) and testing (63) due to GPU limitations. The Liver Tumor Segmentation Benchmark (LiTS17) data set [46] consists of 131 CT scan volumes with various types of tumors. The LiTS17 data set is also divided into $8: 2$ ratio for training and testing in the study. Each CT volume consists of variable number of slices with resolution of $512 \times 512$. 


\section{B. Experimental Setup}

Experiments have been carried out using 3D-QNet and 3DQNet-NonTensor on 3D brain MR volumes collected from the BRATS 2019 dataset of size $240 \times 240$ and on LiTS17 data set of size $512 \times 512$ with MATLAB 2020a. The proposed 3DQNet and 3D-QNet-NonTensor architectures are implemented with the multi-level gray-scale images using distinct multiclass levels $L=4,6$, and 8 characterized by the Vox-QSig activation function. The steepness $\lambda$ is varied in the range 0.23 to 0.24 with step size 0.001 . For FLAIR and T2, it has been observed that in majority of cases, $\lambda=0.232$ and $\mathcal{S}=26(3 \times 3 \times 3$ volume $)$ yield optimal performance. For the other two modalities $T 1$ and T1-CE, $\lambda=0.238$ and $\mathcal{S}=26(3 \times 3 \times 3$ volume $)$ yield optimal performance. On contrary, the LiTS17 CT volume data has performed optimally for $\lambda=0.239$ and $\mathcal{S}=26(3 \times 3 \times 3$ volume $)$. Moreover, the Vox-QSig is guided by four distinct activation schemes $\left(v_{\beta}, v_{\xi}, v_{\zeta}, v_{\kappa}\right)$ [12], [32], [42]. Experiments have also been performed using the 3D-UNet [22] architecture, Deep Voxel-wise Residual Network (VoxResNet) [24], DenseRes-Inception Net (DRINet) [25], and 3D-ESPNet [26] on the BRATS 2019 data set [45] and on the LiTS17 data set [46]. We have trained 3D-UNet [22] and VoxResNet [24] rigorously with the Stochastic Gradient Descent (SGD) algorithm on Caffe library ${ }^{1}$ using a Nvidia DGX Server. The 3D-ESPNet is implemented using Pytorch from the code available in Github ${ }^{2}$ with 100 epochs using adam optimizer with an initial learning rate of 0.01 . The DRINet is also implemented using adam optimizer with an initial learning rate of 0.01 and kernel size of $3 \times 3$. The segmented output images resemble in size with the dimensions of the binary mask and the outcome 1 is considered as tumor region and 0 as background in detecting complete tumor. The pixel by pixel comparison with the manually segmented regions of interest or lesion mask allows evaluating the dice similarity (DS) [16], which is considered as a standard evaluation procedure in automatic medical image segmentation. The evaluation process involves the manually segmented lesion mask as ground truth, and each 2D pixel is predicted as either True Positive $\left(T_{R P}\right)$ or True Negative $\left(T_{R N}\right)$ or False Positive $\left(T_{R N}\right)$ or False Negative $\left(F_{L N}\right)$. The empirical goodness measures [Positive Predictive Value $(P V)$, Sensitivity $(S S)$, Accuracy $(A C)$ and Dice Similarity $(D S)$ [16]] are assessed to evaluate the results.

\section{Experimental Results}

Experiments have been performed in the current setup, and experimental outcomes are reported with the demonstration of numerical and statistical analyses using the proposed 3DQNet, 3D-QNet-NonTensor, 3D-UNet [22], VoxResNet [24], DRINet [25], and 3D-ESPNet [26] on BRATS 2019 data set [45] and LiTS17 data set [46]. It is evident from the experimental data reported in Table I that the proposed 3D-QNet performs optimally for complete brain tumor segmentation of four different modalities of MR volumes (viz. $T 1, T 1-C E$,

\footnotetext{
${ }^{1}$ https://doi.org/10.1145/2647868.2654889

${ }^{2}$ https://github.com/sacmehta/3D-ESPNet
}

$F L A I R$, and $T 2$ ) using the activation guided by 26 -connected heterogeneous voxel intensities $\left(v_{\xi}\right)$ with $L=8$ in comparison with other thresholding schemes under the four evaluation parameters $(A C, D S, P V, S S$ ) [16]. The 3D-QNet segmented brain MR slices collected from two different volumes BRATS19-CBICA-AAG and BRATS19-CBICA-AAB using class level $L=8$ with activation schemes, $v_{\xi}$ are shown in Figures 4 and 5 , respectively. The human expert annotated ground truth slices for all the four different modalities are illustrated in Figure 6. 3D-UNet [22], VoxResNet [24], DRINet [25], 3DESPNet [26], and 3D-QNet-NonTensor segmented brain MR volumes from BRATS19-CBICA-AAG are also demonstrated in the Supplementary Materials. It has been observed from the segmented MR slices that our 3D-QNet is suitable in segmenting the correct position and size of the complete tumor while compared with the ground truth segmentation. However, it is not efficient in mapping the sharp contour of the core and enhanced tumor sub-regions outlined in the annotated slices. Table II presents the quantitative results reported using the proposed 3D-QNet, 3D-UNet [22], VoxResNet [24], DRINet [25], and 3D-ESPNet [26] on evaluating the average accuracy $(A C)$, dice similarity score $(D S)$, positive prediction value $(P V)$, and sensitivity $(S S)$ [16]. It has been observed from the 3DQNet segmented brain MR slices and the results reported in Table II, that optimal segmentation is achieved for FLAIR reported with an average of 0.821 dice score (DS) for FLAIR. The proposed 3D-QNet marginally outperforms the convolutional based architectures (3D-UNet [22], VoxResNet [24], DRINet [25], 3D-ESPNet [26]), and 3D-QNet-NonTensor in predicting complete brain tumor detection. However, it may be noted that our 3D-QNet does not intend to predict the core, enhanced tumor and necrosis sub regions owing to lack of optimization of the parameters in the suggested 3D-QNet. The box plots are also demonstrated in the Supplementary Materials citing the outcome reported in Table II. Moreover, to show the effectiveness of our proposed 3D-QNet over 3D-UNet [22], VoxResNet [24], DRINet [25], and 3DESPNet [26], we have also conducted experiments on the Liver Tumor Segmentation Benchmark (LiTS17) data set [46]. Table III presents the results reported using the proposed 3DQNet, 3D-UNet [22], VoxResNet [24], DRINet [25], and 3DESPNet [26] on LiTS17 data set [46] in detecting complete Liver tumor regions. A sample of segmented Liver tumor using 3D-QNet with manually segmented tumors is shown in Figure 7. It has been observed from the Table III that 3D-QNet has reported with an average Dice Score (DS) of 0.958. Moreover, one-sided two-sample Kolmogorov-Smirnov (KS) [47] test is conducted with significance level $\alpha=0.05$ and it can be summarized from the experimental data reported in Table II and Table III that 3D-QNet is robust in segmenting 3D medical image data. It is interesting to note that despite being characterized by a fully self-supervised quantum learning, the 3D-QNet has shown similar accuracy $(A C)$ and dice similarity $(D S)$ in comparison to 3D-UNet [22], VoxResNet [24], DRINet [25], and 3D-ESPNet [26]. Hence, the performance of the 3D-QNet model on the BRATS 2019 and LiTS17 data sets are statistically significant and offers a promising alternative to selfsupervised deep learning for 3D-medical image segmentation. 
Furthermore, in case of brain MR image segmentation, the number of parameters required in 3D-UNet [22] architecture is $19,069,955$, where as the proposed 3D-QNet architecture employs maximum 2, 995, 200 parameters (considering each voxel as a candidate in a $240 \times 240$ dimensional slice, there are total $240 \times 240 \times 26 \times 2$ connections) in bi-directional propagation.

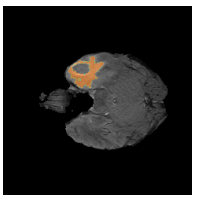

(a) Slice \#44

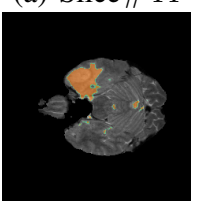

(e) Slice\#44

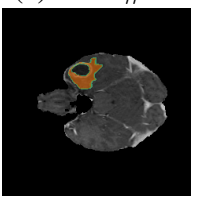

(i) Slice\#44

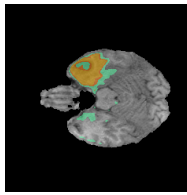

(m) Slice\#44

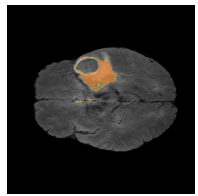

(b) Slice\#59

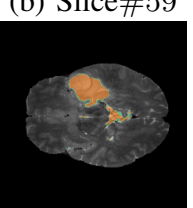

(f) Slice\#59

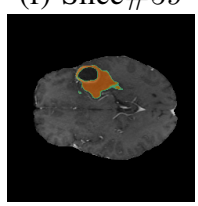

(j) Slice\#59

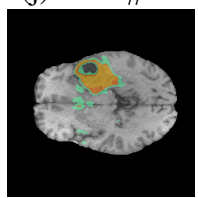

(n) Slice\#59

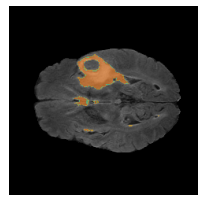

(c) Slice\#64

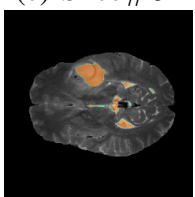

(g) Slice\#64

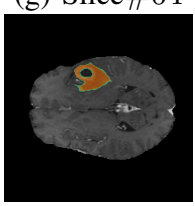

(k) Slice\#64

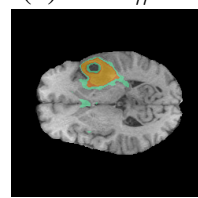

(o) Slice\#64

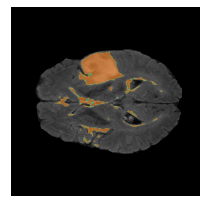

(d) Slice\#69

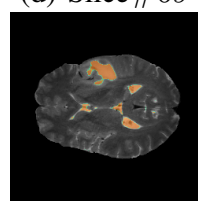

(h) Slice\#69

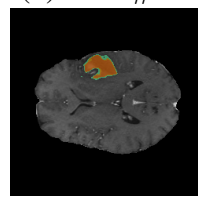

(1) Slice\#69

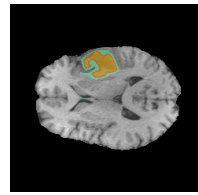

(p) Slice\#69
Fig. 4: 3D-QNet segmented Brain MR volume $(a-d)$ BraTS19-CBICA-AAG-1-flair, $(e-h)$ BraTS19-CBICA-AAG1-t2, $(i-l)$ BraTS19-CBICA-AAG-1-t1ce, $(m-p)$ BraTS19CBICA-AAG-1-t1 from the BRATS 2019 data set [45] (Union of overlapped brown/yellow and green corresponds to a complete tumor (WT) region).

\section{CONCLUSION}

A 3D Quantum-inspired Self-supervised Tensor Neural Network (3D-QNet) architecture characterized by $\mathcal{S}$-connected voxel-wise processing for fully automated semantic segmentation of Brain MR volumes and 3D Liver CT images is presented in this work. Intensive validation using the BRATS 2019 and LiTS17 data sets shows the efficacy of the proposed self-supervised 3D-QNet to promote automatic semantic segmentation of 3D medical images in real-time with minimum human intervention which is still considered as an uphill task in the field of volumetric medical image segmentation. The incorporation of quantum-inspired computing and tensor-based learning in the suggested network model aims to provide faster convergence of the 3D-QNet than its non-tensorized implementation, thereby enabling accurate segmentation results. Despite being a 3D self-supervised network model, 3D-QNet achieved similar dice similarity score on complete tumor detection as deeply supervised 3D-UNet, Vox-ResNet,
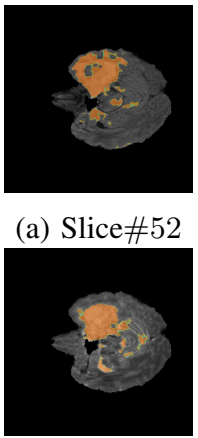

(e) Slice\#52

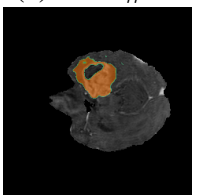

(i) Slice\#52

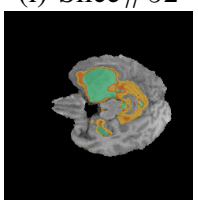

(m) Slice\#52 (a) Slice $\# 52$
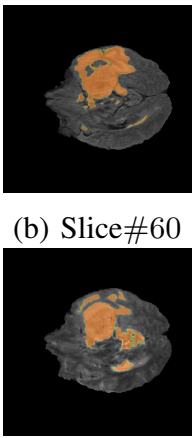

(f) Slice\#60

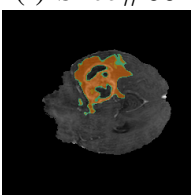

(j) Slice \#60

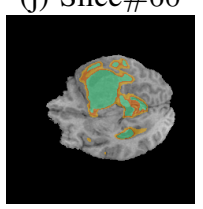

(n) Slice\#60 (b) Slice \#60

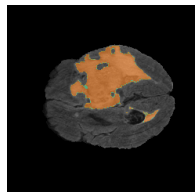

(c) Slice\#71

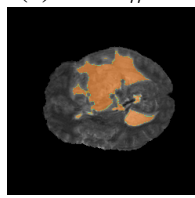

(g) Slice\#71

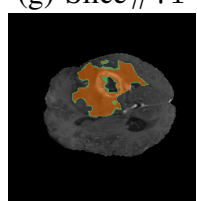

(k) Slice\#71

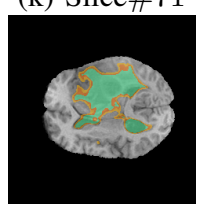

(o) Slice\#71

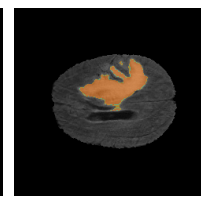

(d) Slice\#95

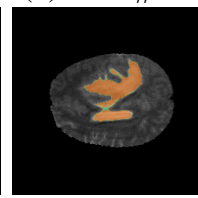

(h) Slice\#95

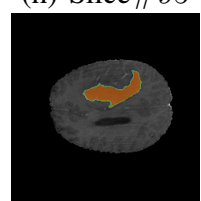

(1) Slice $\# 95$

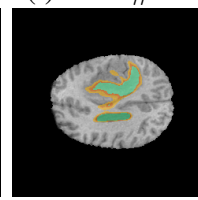

(p) Slice\#95
Fig. 5: 3D-QNet segmented Brain MR volume $(a-d)$ BraTS19-CBICA-AAB-1-flair, $(e-h)$ BraTS19-CBICA-AAB1-t2, $(i-l)$ BraTS19-CBICA-AAB-1-t1ce, $(m-p)$ BraTS19CBICA-AAB-1-t1 from the BRATS 2019 data set [45] (Union of overlapped brown/yellow and green corresponds to a complete tumor (WT) region).

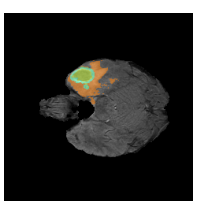

(a) Slice \#44

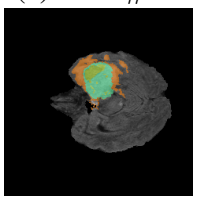

(e) Slice\#52

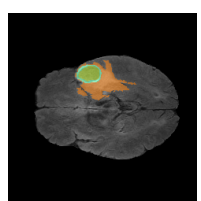

(b) Slice\#59

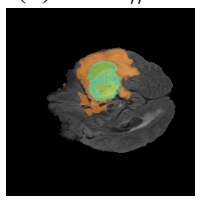

(f) Slice\#60

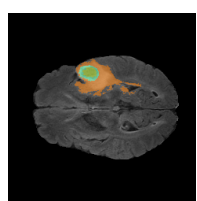

(c) Slice\#64

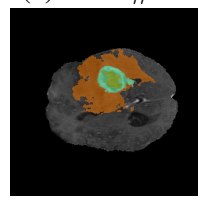

(g) Slice\#71

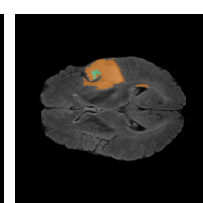

(d) Slice\#69

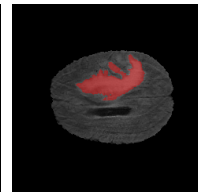

(h) Slice\#95
Fig. 6: Annotated Brain MR volume $(a-d)$ BraTS19-CBICAAAG-1-seg, $(e-h)$ BraTS19-CBICA-AAB-1-seg from the BRATS 2019 data set [45] (Complete tumor (WT) region comprises a union of brown, light green and green yellow, core tumor (TC) is the union of light green and green yellow, and green yellow corresponds to the tumor enhancing (TE)). 
TABLE I: Results obtained using proposed 3D-QNet for complete tumor (WT) segmentation on BraTS19-CBICA-AAG-1flair-slice \#69

\begin{tabular}{|c|c|c|c|c|c|c|c|c|c|c|c|c|c|c|c|c|c|}
\hline \multirow[t]{2}{*}{ Level } & \multirow[t]{2}{*}{ Modality } & \multicolumn{4}{|c|}{$\frac{\mathbf{A C} \overline{\bar{T}}_{R P}+T_{R N}}{\frac{T_{R P}+F_{L P}+T_{R N}+F_{L N}}{T_{R}}}$} & \multicolumn{3}{|c|}{$\frac{\text { DS } \overline{\overline{2 T}}_{R P}}{\frac{2 T_{R P}+F_{L P}+F_{L N}}{2}}$} & & \multicolumn{3}{|c|}{$\begin{array}{c}\mathbf{P V}= \\
\frac{T_{R P}}{T_{R P}+F_{L P}}\end{array}$} & & \multicolumn{2}{|c|}{$\begin{array}{c}\mathbf{S S}= \\
\frac{T_{R P}}{T_{R P}+F_{L N}}\end{array}$} & \multirow[b]{2}{*}{$v_{\xi}$} & \multirow[b]{2}{*}{$v_{\kappa}$} \\
\hline & & $v_{\beta}$ & $v_{\chi}$ & $v_{\xi}$ & $v_{\kappa}$ & $v_{\beta}$ & $v_{\chi}$ & $v_{\xi}$ & $v_{\kappa}$ & $v_{\beta}$ & $v_{\chi}$ & $v_{\xi}$ & $v_{\kappa}$ & $v_{\beta}$ & $v_{\chi}$ & & \\
\hline \multirow{4}{*}{$L=4$} & $T 1$ & 0.99 & $\frac{\chi}{0.99}$ & 0.99 & 0.99 & 0.62 & 0.80 & 0.80 & 0.79 & 0.44 & 0.66 & 0.65 & 0.65 & 0.99 & 0.99 & 0.99 & 0.99 \\
\hline & $T 1-C E$ & 0.99 & 0.99 & 0.99 & 0.99 & 0.79 & 0.80 & 0.79 & 0.80 & 0.66 & 0.66 & 0.66 & 0.66 & 0.99 & 0.99 & 0.99 & 0.99 \\
\hline & $F L A I R$ & 0.99 & 0.99 & 0.99 & 0.99 & 0.62 & 0.80 & 0.80 & 0.79 & 0.44 & 0.66 & 0.66 & 0.65 & 0.99 & 0.99 & 0.99 & 0.99 \\
\hline & $T 2$ & 0.99 & 0.99 & 0.99 & 0.99 & 0.62 & 0.80 & 0.80 & 0.79 & 0.44 & 0.66 & 0.66 & 0.65 & 0.99 & 0.99 & 0.99 & 0.99 \\
\hline \multirow[b]{3}{*}{$L=6$} & $T 1$ & 0.99 & 0.99 & 0.99 & 0.99 & 0.62 & 0.80 & 0.80 & 0.79 & 0.44 & 0.66 & 0.66 & 0.65 & 0.99 & 0.99 & 0.99 & 0.99 \\
\hline & $T 1-C E$ & 0.99 & 0.99 & 0.99 & 0.99 & 0.62 & 0.80 & 0.80 & 0.79 & 0.44 & 0.66 & 0.66 & 0.65 & 0.99 & 0.99 & 0.99 & 0.99 \\
\hline & $F L A I R$ & 0.99 & 0.99 & 0.99 & 0.99 & 0.62 & 0.80 & 0.80 & 0.79 & 0.44 & 0.66 & 0.66 & 0.65 & 0.99 & 0.99 & 0.99 & 0.99 \\
\hline \multirow{4}{*}{$L=8$} & $T 1$ & 0.99 & 0.99 & 0.99 & 0.99 & 0.82 & 0.82 & 0.82 & 0.81 & 0.70 & 0.69 & 0.69 & 0.68 & 0.99 & 0.99 & 0.99 & 0.99 \\
\hline & $T 1-C E$ & 0.99 & 0.99 & 0.99 & 0.99 & 0.81 & 0.81 & 0.81 & 0.81 & 0.68 & 0.68 & 0.68 & 0.68 & 0.99 & 0.99 & 0.99 & 0.99 \\
\hline & $F L A I R$ & 0.99 & 0.99 & 0.99 & 0.99 & 0.84 & 0.84 & 0.84 & 0.84 & 0.73 & 0.73 & 0.73 & 0.72 & 0.99 & 0.99 & 0.98 & 0.98 \\
\hline & $T 2$ & 0.99 & 0.99 & 0.99 & 0.99 & 0.82 & 0.82 & 0.82 & 0.82 & 0.69 & 0.69 & 0.70 & 0.70 & 0.99 & 0.99 & 0.99 & 0.99 \\
\hline
\end{tabular}

TABLE II: Comparative analysis of proposed 3D-QNet with 3D-QNet-NonTensor, 3D-UNet [22], VoxResNet [24], DRINet [25], and 3D-ESPNet [26] [The bold values reflect evaluation metrics with significance level $\alpha=0.05$ conducted with one sided two sample KS test [47]]

\begin{tabular}{llllll}
\hline Methods & Modality & AC & DS & PV & SS \\
\hline \multirow{3}{*}{ 3D-UNet [22] } & $T 1$ & 0.990 & 0.811 & 0.736 & 0.941 \\
& $T 1-C E$ & 0.990 & 0.807 & 0.732 & 0.938 \\
& $F L A I R$ & $\mathbf{0 . 9 9 2}$ & $\mathbf{0 . 8 2 3}$ & 0.737 & 0.943 \\
& $T 2$ & 0.989 & 0.812 & 0.735 & 0.944 \\
\hline \multirow{3}{*}{ VoxResNet [24] } & $T 1$ & 0.990 & 0.810 & 0.737 & 0.937 \\
& $T 1-C E$ & 0.989 & 0.813 & 0.732 & 0.943 \\
& $F L A I R$ & $\mathbf{0 . 9 9 1}$ & $\mathbf{0 . 8 2 2}$ & $\mathbf{0 . 7 5 1}$ & 0.942 \\
& $T 2$ & 0.990 & 0.807 & 0.729 & 0.944 \\
\hline \multirow{3}{*}{ DRINet [25] } & $T 1$ & 0.989 & 0.793 & 0.701 & 0.958 \\
& $T 1-C E$ & 0.988 & 0.800 & 0.711 & 0.959 \\
& $F L A I R$ & 0.989 & 0.805 & 0.708 & $\mathbf{0 . 9 6 9}$ \\
3D-ESPNet $[26]$ & $T 2$ & 0.987 & 0.789 & 0.700 & 0.958 \\
\hline \multirow{3}{*}{ 3D-QNet } & $T 1$ & 0.989 & 0.801 & 0.709 & 0.961 \\
& $T 1-C E$ & 0.989 & 0.813 & 0.721 & $\mathbf{0 . 9 6 6}$ \\
& $F L A I R$ & 0.989 & 0.800 & 0.715 & 0.959 \\
& $T 2$ & 0.988 & 0.802 & 0.714 & 0.957 \\
\hline \multirow{3}{*}{ 3D-QNet-NonTensor } & $T 1$ & 0.989 & 0.801 & 0.736 & $\mathbf{0 . 9 6 5}$ \\
& $T 1-C E$ & 0.989 & 0.811 & 0.740 & 0.957 \\
& $F L A I R$ & $\mathbf{0 . 9 9 1}$ & $\mathbf{0 . 8 2 1}$ & $\mathbf{0 . 7 5 1}$ & 0.957 \\
& $T 2$ & 0.990 & 0.814 & 0.736 & 0.960 \\
\hline & $T 2$ & 0.987 & 0.776 & 0.678 & 0.959 \\
& & 0.987 & 0.772 & 0.678 & 0.958 \\
& & 0.989 & 0.786 & 0.697 & 0.956 \\
& & 0.988 & 0.788 & 0.696 & 0.957 \\
\hline
\end{tabular}

TABLE III: Comparative Results on Liver Segmentation using proposed 3D-QNet, 3D-UNet [22], VoxResNet [24], DRINet [25], and 3D-ESPNet [26] [The bold values reflect evaluation metrics with significance level $\alpha=0.05$ conducted with one sided two sample KS test [47]]

\begin{tabular}{lllll}
\hline Methods & AC & DS & PV & SS \\
\hline VoxResNet [24] & $\mathbf{0 . 9 9 1}$ & $\mathbf{0 . 9 6 1}$ & 0.798 & 0.973 \\
\hline 3D-QNet & $\mathbf{0 . 9 8 9}$ & $\mathbf{0 . 9 5 8}$ & 0.801 & 0.965 \\
\hline 3D-UNet [22] & $\mathbf{0 . 9 9 1}$ & $\mathbf{0 . 9 5 9}$ & $\mathbf{0 . 8 3 0}$ & 0.964 \\
\hline DRINet [25] & 0.980 & 0.951 & 0.802 & $\mathbf{0 . 9 8 8}$ \\
\hline 3D-ESPNet [26] & 0.987 & 0.943 & 0.798 & 0.961 \\
\hline
\end{tabular}

DRINet and ESPNet, thus promoting self-supervised network learning for volumetric segmentation of medical images. In principle, the proposed 3D-QNet is a general self-supervised network architecture which can be extended to many other 3D medical image segmentation avenues, where the segmented

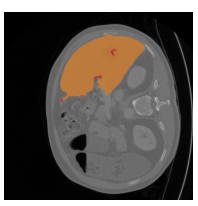

(a) Slice\#172

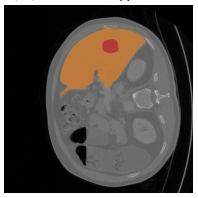

(e) Slice\#172
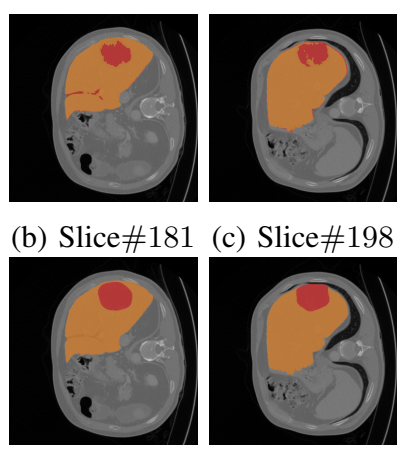

(f) Slice\#181

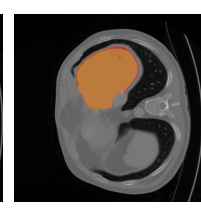

(d) Slice\#206

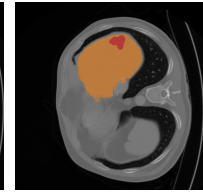

(g) Slice\#198 (h) Slice\#206
Fig. 7: $(a-d)$ 3D-QNet segmented volumetric Liver segmented image slices (scan\#64), $(e-h)$ manually segmented image slices (scan\#64) from data set [46] (Union of overlapped brown and red corresponds to a complete tumor (WT) region).

annotations are limited. Moreover, our self-supervised model can be employed in any application setting (eg. medical IoT devices) right away where, the deep learning models face serious obstacles. However, the 3D-QNet fails to yield optimal outcome for multi-level segmentation on the BRATS 2019 data set. The authors are currently engaged in extending the 3DQNet architecture by up-scaling the intermediate volumetric features in the network and optimizing its hyper-parameters to yield optimal segmentation outcome.

\section{APPENDIX}

\section{A. Convergence Analysis of $3 D-Q N e t$}

Le us assume the optimal phase angles at depth $d$ for the weighted matrix and the activation are denoted as $\overline{\omega^{d}}$ and $\overline{\vartheta^{d}}$, respectively. Now, consider

$$
\begin{aligned}
& \mathcal{W}^{l, d}=\omega^{l, d}-\overline{\omega^{d}} \\
& \mathcal{V}^{l, d}=\vartheta^{l, d}-\overline{\vartheta^{d}}
\end{aligned}
$$

and

$$
\begin{gathered}
\mathcal{D}^{l, d}=\omega^{l+1, d}-\omega^{l, d}=\mathcal{W}^{l+1, d}-\mathcal{W}^{l, d} \\
\mathcal{P}^{l, d}=\vartheta^{l+1, d}-\vartheta^{l, d}=\mathcal{V}^{l+1, d}-\mathcal{V}^{l, d}
\end{gathered}
$$

The loss function $\zeta\left(\omega^{l, d}, \vartheta^{l, d}\right)$ is differentiable with respect to $\omega^{l, d}$ and $\vartheta^{l, d}$ as

$$
\begin{array}{r}
\frac{\partial \zeta\left(\omega^{l, d}, \vartheta^{l, d}\right)}{\partial \omega_{i j}^{l, d}}=\frac{2}{\mathcal{N}} \sum_{i=1}^{\mathcal{N}} \sum_{j=1}^{\mathcal{S}} \Delta \varphi_{i j}^{l, d}\left(\omega_{i j}^{l, d}, \vartheta_{j}^{l, d}\right) \\
{\left[\frac{\partial \varphi_{i j}^{l+1, d}\left(\omega_{i j}^{l+1, d}, \vartheta_{j}^{l+1, d}\right)}{\partial \omega_{i j}^{l+1, d}}-\frac{\partial \varphi_{i j}^{l, d}\left(\omega_{i j}^{l, d}, \vartheta_{j}^{l, d}\right)}{\partial \omega_{i j}^{l, d}}\right]}
\end{array}
$$




$$
\begin{array}{r}
\frac{\partial \zeta\left(\omega^{l, d}, \vartheta^{l, d}\right)}{\partial \vartheta_{j}^{l, d}}=\frac{2}{\mathcal{N}} \sum_{i=1}^{\mathcal{N}} \sum_{j=1}^{\mathcal{S}} \Delta \varphi_{i j}^{l, d}\left(\omega_{i j}^{l, d}, \vartheta_{j}^{l, d}\right) \\
{\left[\frac{\partial \varphi_{i j}^{l+1, d}\left(\omega_{i j}^{l+1, d}, \vartheta_{j}^{l+1, d}\right)}{\partial \vartheta_{j}^{l+1, d}}-\frac{\partial \varphi_{i j}^{l, d}\left(\omega_{i j}^{l, d}, \vartheta_{j}^{l, d}\right)}{\partial \vartheta_{j}^{l, d}}\right]}
\end{array}
$$

where,

$$
\triangle \varphi_{i j}^{l, d}\left(\omega_{i j}^{l, d} \vartheta_{j}\right)=\left|\varphi_{i j}^{l+1, d}\left(\omega_{i j}^{l+1, d}, \vartheta_{j}^{l+1, d}\right)-\varphi_{i j}^{l, d}\left(\omega_{i j}^{l, d}, \vartheta_{j}^{l, d}\right)\right|
$$

The following equations evaluate the change in phase or angles $(\triangle \omega$ and $\triangle \alpha$ ) of the rotation gates as

$$
\begin{aligned}
& \triangle \omega_{i j}^{l, d}=-\rho_{i j}^{l, d}\left\{\frac{\partial \varphi\left(\omega^{l, d}, \vartheta^{l, d}\right)}{\partial \omega_{i j}^{l, d}} \varphi\left(\omega^{l, d}, \vartheta^{l, d}\right)\right\}^{\frac{1}{\tau}} \\
& \left.\triangle \vartheta_{j}^{l, d}=-\kappa_{j}^{l, d}\left\{\frac{\partial \varphi\left(\omega^{l, d}, \vartheta^{l, d}\right)}{\partial \vartheta_{i}^{l, d}} \varphi\left(\omega^{l, d}, \vartheta^{l, d}\right)\right)\right\}^{\frac{1}{\tau}}
\end{aligned}
$$

where, $\rho_{i j}$ and $\kappa_{j}$ refer to the learning rates for the adjustments of weights and activation, respectively and are evaluated as

$$
\begin{aligned}
\rho_{i j}^{l, d}=\mathcal{X}_{i}^{l, d}-\mathcal{X}_{i j}^{l, d} \forall j & =1,2 \ldots 8 \\
\kappa_{j}^{l, d}=\left(\sum_{j} \mathcal{X}_{i, j}^{l, d}\right) \forall j & =1,2 \ldots 8
\end{aligned}
$$

The sequences of $\left\{\omega^{l, d}\right\}$ and $\left\{\vartheta^{l, d}\right\}$ converge super-linearly subject to the following conditions [13].

$$
\lim _{l \rightarrow \infty} \frac{\left\|\omega^{l+1, d}-\bar{\omega}^{d}\right\|}{\left\|\omega^{l, d}-\bar{\omega}^{d}\right\|} \leq 1
$$

and

$$
\left\|\mathcal{W}^{l+1, d}\right\|=O\left\|\mathcal{D}^{l, d}\right\|
$$

Also,

$$
\lim _{l \rightarrow \infty} \frac{\left\|\vartheta^{l+1, d}-\bar{\vartheta}^{d}\right\|}{\left\|\vartheta^{l}-\bar{\vartheta}^{d}\right\|} \leq 1
$$

and

$$
\left\|\mathcal{V}^{l+1, d}\right\|=O\left\|\mathcal{P}^{l, d}\right\|
$$

The convergence of the sequence $\left\{\omega^{l, d}\right\}$ according to $L$-Lipschitz continuity is illustrated as [48]

$$
\begin{array}{r}
\zeta\left(\omega^{l+1, d}\right) \leq \zeta\left(\omega^{l, d}\right)+\left\langle\nabla_{\omega} \zeta\left(\omega^{l, d}\right), \omega^{l+1, d}-\omega^{l, d}\right\rangle+\frac{L}{2}\left\|\omega^{l+1, d}-\omega^{l, d}\right\|^{2} \\
=\zeta\left(\omega^{l, d}\right)+\left\langle\nabla_{\omega} \zeta\left(\omega^{l, d}\right)-\rho \nabla_{\omega} \zeta\left(\omega^{l, d}\right)\right\rangle+\frac{L}{2}\left\|-\rho \nabla_{\omega} \zeta\left(\omega^{l, d}\right)\right\|^{2} \\
=\zeta\left(\omega^{l, d}\right)-\rho\left\|\nabla_{\omega} \zeta\left(\omega^{l, d}\right)\right\|^{2}+\rho^{2} \frac{L}{2}\left\|\nabla_{\omega} \zeta\left(\omega^{l, d}\right)\right\|^{2} \\
=\zeta\left(\omega^{l, d}\right)-\rho\left(1-\rho \frac{L}{2}\right)\left\|\nabla_{\omega} \zeta\left(\omega^{l, d}\right)\right\|^{2} \\
\leq \zeta\left(\omega^{l, d}\right)-\frac{\rho}{2}\left\|\nabla_{\omega} \zeta\left(\omega^{l, d}\right)\right\|^{2}\left(\text { Assuming, } \rho \in\left(0, \frac{1}{L}\right]\right) \\
\leq \zeta\left(\overline{\omega^{d}}\right)+\left\langle\nabla_{\omega} \zeta\left(\omega^{l, d}\right), \omega^{l, d}-\overline{\omega^{d}}\right\rangle-\frac{\rho}{2}\left\|\nabla_{\omega} \zeta\left(\omega^{l, d}\right)\right\|^{2},(\zeta i s \text { convex }) \\
=\zeta\left(\overline{\omega^{d}}\right)+\left\langle\nabla_{\omega} \zeta\left(\omega^{l, d}\right), \omega^{l, d}-\overline{\omega^{d}}\right\rangle-\frac{\rho}{2}\left\|\nabla_{\omega} \zeta\left(\omega^{l, d}\right)\right\|^{2}+ \\
\frac{1}{2 \rho}\left(\left\|\omega^{l, d}-\overline{\omega^{d}}\right\|^{2}-\left\|\omega^{l, d}-\overline{\omega^{d}}\right\|^{2}\right) \\
=\zeta\left(\overline{\omega^{d}}\right)+\frac{1}{2 \rho}\left(\left\|\omega^{l, d}-\overline{\omega^{d}}\right\|^{2}-\left(\left.\left\|\omega^{l, d}\right\|\right|^{2}-2\left\langle\omega^{l, d}, \overline{\omega^{d}}\right\rangle+\right.\right. \\
\left.\left.\left\|\overline{\omega^{d}}\right\|^{2}-2 \rho\left\langle\nabla_{\omega} \zeta\left(\omega^{l, d}\right), \omega^{l, d}-\overline{\omega^{d}}\right\rangle+\rho^{2}\left\|\nabla_{\omega} \zeta\left(\omega^{l, d}\right)\right\|^{2}\right)\right) \\
=\zeta\left(\overline{\omega^{d}}\right)+\frac{1}{2 \rho}\left(\left\|\omega^{l, d}\right\|-\overline{\omega^{d}} \|^{2}-\left(\left\|\omega^{l, d}-\rho \nabla_{\omega} \zeta\left(\omega^{l, d}\right)\right\|^{2}-\right.\right. \\
=\zeta\left(\overline{\omega^{d}}\right)+\frac{1}{2 \rho}\left(\left\|\omega^{l, d}-\overline{\omega^{d}}\right\|^{2}-\left\|\omega^{l+1, d}-\overline{\omega^{d}}\right\|^{2}\right) \\
\therefore \zeta\left(\omega^{l+1, d}\right)-\zeta\left(\overline{\omega^{d}}\right) \leq \frac{1}{2 \rho}\left(\left\|\omega^{l, d}-\overline{\omega^{d}}\right\|^{2}-\left\|\omega^{l+1, d}-\overline{\omega^{d}}\right\|^{2}\right)
\end{array}
$$

Similarly, it can also be shown that

$$
\zeta\left(\vartheta^{l+1, d}\right)-\zeta\left(\overline{\vartheta^{l, d}}\right) \leq \frac{1}{2 \rho}\left(\left\|\vartheta^{l+1, d}-\overline{\vartheta^{d}}\right\|^{2}-\left\|\vartheta^{l+1, d}-\overline{\vartheta^{d}}\right\|^{2}\right)
$$

Now, according to Thaler formula

$$
\begin{array}{r}
\zeta\left(\omega^{l+1, d}, \vartheta^{l+1, d}\right)-\zeta\left(\omega^{l, d}, \vartheta^{l, d}\right)= \\
{\left[\begin{array}{ll}
\triangle \omega_{i j}^{l, d} & \triangle \vartheta_{j}^{l, d}
\end{array}\right]\left[\begin{array}{c}
\frac{\partial \zeta\left(\omega^{l, d}, \vartheta^{l, d}\right)}{\partial \omega_{i j}^{l, d}} \\
\frac{\partial \zeta\left(\omega^{l, d, \vartheta^{l, d}}\right.}{\partial \vartheta_{j}^{l, d}}
\end{array}\right]+O\left[\begin{array}{ll}
\| \Delta \omega_{i j}^{l, d} & \triangle \vartheta_{i}^{l, d} \|
\end{array}\right]}
\end{array}
$$

$$
\approx\left[\left\{-\rho_{i j}^{l, d} \frac{\partial \zeta\left(\omega^{l, d}, \vartheta^{l, d}\right)}{\partial \omega_{i j}^{l, d}}\right\}^{2}+\left\{-\kappa_{j}^{l, d} \frac{\partial \zeta\left(\omega^{l, d}, \vartheta^{l, d}\right)}{\partial \vartheta_{j}^{l, d}}\right\}^{2}\right]\left\{\zeta\left(\omega^{l, d}, \vartheta^{l, d}\right)\right\}^{\frac{1}{\iota}}
$$

It is obvious that $\left(\zeta\left(\omega^{l+1, d}, \alpha^{l+1, d}\right)-\zeta\left(\omega^{l, d}, \vartheta^{l, d}\right)\right) \leq 0$ and the sequences of $\left\{\omega^{l, d}\right\}$ and $\left\{\vartheta^{l, d}\right\}$ are monotonically decreasing as

$$
\lim _{l \rightarrow \infty} \zeta\left(\omega^{l, d}, \vartheta^{l, d}\right)=\left(\bar{\omega}^{d}, \bar{\vartheta}^{d}\right)
$$

and

$$
\lim _{l \rightarrow \infty} \frac{\left\|\zeta\left(\omega^{l+1, d}, \vartheta^{l+1, d}\right)-\left(\bar{\omega}^{d}, \bar{\vartheta}^{d}\right)\right\|}{\left\|\zeta\left(\omega^{l, d}, \vartheta^{l, d}\right)-\left(\bar{\omega}^{d}, \bar{\vartheta}^{d}\right)\right\|} \leq 1
$$

\section{B. Code Availability}

3D-QNet implementation is made available in GitHub: https://github.com/konar1987/ 3D-QNet for brain volume image segmentation with few samples, tailored and tested for FLAIR and T2 from the BRATS 2019 data set [45].

\section{REFERENCES}

[1] G. Litjens et al., "A survey on deep learning in medical image analysis," Medical Image Analysis, vol. 42, pp. 60—88, 2017. DOI: https://doi.org/ 10.1016/j.media.2017.07.005.

[2] M. Huang, W. Yang, Y. Wu, J. Jiang, W. Chen, and Q. Feng, "Brain Tumor Segmentation Based on Local Independent Projection-Based Classification," IEEE Transactions on Biomedical Engineering, vol. 61, no. 10, pp. 2633-2645, 2014, DOI: 10.1109/TBME.2014.2325410.

[3] Z. Wu, K. D. Paulsen, and J. M. Sullivan, "Adaptive model initialization and deformation for automatic segmentation of T1-weighted brain MRI data", IEEE Transactions on Biomedical Engineering, vol. 52, no. 6, pp. 1128-1131, 2005, DOI: 10.1109/TBME.2005.846709.

[4] M. Chung, J. Lee, M. Lee, J. Lee, Y-G. Shin, "Deeply self-supervised contour embedded neural network applied to liver segmentation," Computer Methods and Programs in Biomedicine, vol. 192, pp. 105447, 2020, https://doi.org/10.1016/j.cmpb.2020.105447.

[5] F. Milletari, N. Navab, and S. A. Ahmadi, "V-Net: Fully Convolutional Neural Networks for Volumetric Medical Image Segmentation" 2016 Fourth International Conference on 3D Vision (3DV), pp. 565--571, 2016 DOI:10.1109/3DV.2016.79.

[6] O. Ronneberger, P. Fischer, T. Brox, "U-Net: convolutional networks for biomedical image segmentation. International Conference on Medical Image Computing and Computer-Assisted Intervention (MICCAI 2015), vol. 9351, pp. 234--241. 2016. DOI: https://doi.org/10.1007/ 978-3-319-46723-8_49.

[7] Q. Doua, L. Yua, H. Chena, Y.Jina, X. Yanga, J. Q. Pheng, and A. Heng, "3D deeply supervised network for automated segmentation of volumetric medical images," Medical Image Analysis, vol. 41, pp. 40-54, 2017, DOI: https://doi.org/10.1016/j.media.2017.05.001.

[8] V. Gandhi, G. Prasad, D. Coyle, L. Behera, and T. M. McGinnity, "Quantum neural network-based EEG filtering for a brain-computer interface," IEEE Transaction on Neural Network and Learning Systems, vol. 25 , no. 2, pp. 278--288, 2014, DOI: 10.1109/TNNLS.2013.2274436.

[9] P. Li, H. Xiao, F. Shang, X. Tong, X. Li, and M. Cao, "A hybrid quantuminspired neural networks with sequence inputs," Neurocomputing, vol. 117, pp. 81--90, 2013, DOI: https://doi.org/10.1016/j.neucom.2013.01. 029.

[10] N. Masuyama, C. K. Loo, M. Seera, and N. Kubota, "Quantum-Inspired Multidirectional Associative Memory With a Self-Convergent Iterative Learning," IEEE Transaction on Neural Network and Learning Systems, vol. 29, no. 4, pp. 1058-1068, 2018, DOI: 10.1109/TNNLS.2017. 2653114.

[11] S. Bhattacharyya, P. Pal and S. Bhowmick, "Binary Image Denoising Using a Quantum Multilayer Self Organizing Neural Network," Applied Soft Computing, vol. 24, pp. 717-729, 2014, DOI: https://doi.org/10.1016/ j.asoc.2014.08.027.

[12] D. Konar, S. Bhattacharyya, T. K. Gandhi and B. K. Panigrahi, "A quantum-inspired self-supervised Network model for automatic segmentation of brain MR images," Applied Soft Computing, vol. 93, 2020, DOI: https://doi.org/10.1016/j.asoc.2020.106348.

[13] D. Konar, S. Bhattacharyya, B. K. Panigrahi and E. C. Behrman, "QutritInspired Fully Self-Supervised Shallow Quantum Learning Network for Brain Tumor Segmentation," IEEE Transactions on Neural Networks and Learning Systems, May, 2021, DOI: 10.1109/TNNLS.2021.3077188.

[14] M. Havaei et al., "Brain tumor segmentation with deep neural networks," Medical Image Analysis, vol. 35, pp. 18-31, 2017, DOI: https://doi.org/ 10.1016/j.media.2016. 
[15] E. Shelhamer, J. Long, and T. Darrell, "Fully convolutional networks for semantic segmentation," IEEE Transactions on Pattern Analysis and Machine Intelligence, vol. 39, no. 4, pp. 640-651, 2017, DOI: 10.1109/ TPAMI.2016.2572683.

[16] S. Pereira, A. Pinto, V. Alves, and C. A. Silva, "Brain Tumor Segmentation Using Convolutional Neural Networks in MRI Images," IEEE Transactions on Medical Imaging, vol.35, no. 5, 2016, DOI: 10.1109/ TMI.2016.2538465.

[17] P. Moeskops, M. A. Viergever, A. M. Mendrik, L. S. Vries, M. J. N. L. Benders and I. Išgum, "Automatic Segmentation of MR Brain Images With a Convolutional Neural Network,"IEEE Transactions on Medical Imaging, vol.35, no.5, 2016, DOI: 10.1109/TMI.2016.2548501.

[18] G. Wang et al., "DeepIGeoS: A Deep Interactive Geodesic Framework for Medical Image Segmentation," IEEE Transactions on Pattern Analysis and Machine Intelligence, vol. 41, no. 7, pp. 1559-1572, 2019, DOI: 10.1109/TPAMI.2018.2840695.

[19] S. Banerjee, L. Magee, D. Wang et al., "Semantic segmentation of microscopic neuroanatomical data by combining topological priors with encoder-decoder deep networks," Nature Machine Intelligence, vol. 2, pp. 585-594, 2020, DOI: https://doi.org/10.1038/s42256-020-0227-9.

[20] K. Kamnitsas, "Efficient multi-scale 3D CNN with fully connected CRF for accurate brain lesion segmentation," Medical Image Analysis, vol. 36, pp. 61-78, 2017, DOI: https://doi.org/10.1016/j.media.2016.10.004

[21] Q. Dou, H. Chen, L. Yu, L. Zhao, J. Qin, D. Wang, V. C. Mok, L. Shi, and P. A. Heng. "Automatic Detection of Cerebral Microbleeds From MR Images via 3D Convolutional Neural Networks, IEEE Transactions on Medical Imaging, vol. 35, no. 5, pp. 1182--1195, 2016, DOI: 10.1109/ TMI.2016.2528129.

[22] Ö. Çiçek, A. Abdulkadir, S. S. Lienkamp, T. Brox, and O. Ronneberger, "3D U-Net: Learning Dense Volumetric Segmentation from Sparse Annotation," International Conference on Medical Image Computing and Computer-Assisted Intervention (MICCAI 2016), pp. 424--432, 2016, DOI: https://doi.org/10.1007/978-3-319-46723-8_49.

[23] A. Brebisson, and G. Montana, "Deep Neural Networks for Anatomical Brain Segmentation," In: Proceedings of the IEEE Conference on Computer Vision and Pattern Recognition Workshops, pp. 20--28, 2015, DOI: 10.1109/CVPRW.2015.7301312.

[24] H. Chen, Q. Dou, L. Yu, J. Qin, and P. A. Heng, 2017. "VoxResNet: Deep voxelwise residual networks for brain segmentation from 3D MR images," NeuroImage, vol. 170, pp. 446-455, 2017, DOI: https://doi.org/ 10.1016/j.neuroimage.2017.04.041.

[25] L.Chen, P. Bentley, K. Mori, K. Misawa, M. Fujiwara, and D. Rueckert, "DRINet for Medical Image Segmentation," IEEE Transaction on Medical Imaging, vol. 37, no. 11, 2018, DOI: 10.1109/TMI.2018.2835303.

[26] N. Nuechterlein and S. Mehta, "3D-ESPNet with Pyramidal Refinement for Volumetric Brain Tumor Image Segmentation," International MICCAI Brainlesion Workshop (BrainLes 2018), pp. 245-253, 2018, DOI: https: //doi.org/10.1007/978-3-030-11726-9_22.

[27] G. Wang et al., "Interactive Medical Image Segmentation Using Deep Learning With Image-Specific Fine Tuning," IEEE Transactions on Medical Imaging, vol. 37, no. 7, 2018, DOI: 10.1109/TMI.2018.2791721.

[28] N. Tajbakhsh et al., "Convolutional Neural Networks for Medical Image Analysis: Full Training or Fine Tuning?," IEEE Transactions on Medical Imaging, vol. 35, no. 5, pp. 1299-1312, 2016, DOI: 10.1109/TMI.2016. 2535302.

[29] X. Zhuang, Y. Li, Y. Hu, K. Ma, Y. Yang, and Y. Zheng, "Self-supervised Feature Learning for 3D Medical Images by Playing a Rubik's Cube," International Conference on Medical Image Computing and ComputerAssisted Intervention (MICCAI 2019), pp. 420-428, https://doi.org/10. 1007/978-3-030-32251-9_46.

[30] M. Blendowski, H. Nickisch, M. P. Heinrich, "How to Learn from Unlabeled Volume Data: Self-supervised 3D Context Feature Learning," Proc. Medical Image Computing and Computer Assisted Intervention - MICCAI 2019, vol 11769, 2019, https://doi.org/10.1007/978-3-030-32226-7 72.

[31] A. Ghosh, N. R. Pal, and S. K. Pal, "Self organization for object extraction using a multilayer neural network and fuzziness measures," IEEE Transactions on Fuzzy Systems, vol. 1, no.1, pp. 54-68, 1993, DOI: 10.1109/TFUZZ.1993.390285.

[32] S. Bhattacharyya, P. Dutta and U. Maulik, "A parallel bi-directional self-organizing neural network (PBDSONN) architecture for color image extraction and segmentation," Neurocomputing, vol. 86, pp. 1-23, 2012, DOI: https://doi.org/10.1016/j.neucom.2011.11.025.

[33] G. Purushothaman, N. B. Karayiannis, "Quantum neural networks (QNNs): inherently fuzzy feedforward neural networks," IEEE Transactions on Neural Networks, vol. 8 , no. 3, 1997, DOI: 10.1109/72.572106.
[34] T. C. Lu, G. R. Yu, and J. C. Juang, "Quantum-based algorithm for optimizing artificial neural networks," IEEE Transaction on Neural Network and Learning Systems, vol. 24, no. 8, pp. 1266-1278, 2013, DOI: $10.1109 /$ TNNLS.2013.2249089.

[35] Nam-H. Nguyen ; E. C. Behrman, A. Moustafa, J. E. Steck, "Benchmarking Neural Networks For Quantum Computations," IEEE Transactions on Neural Networks and Learning Systems, pp. 1-10, 2019, DOI: $10.1109 /$ TNNLS.2019.2933394.

[36] E. M. Stoudenmire, and D. J. Schwab, "Supervised Learning with Quantum-Inspired Tensor Networks," https://www.arxiv-vanity.com/ papers/1605.05775/, 2020.

[37] D. Konar, S. Bhattacharya, B. K. Panigrahi, K. Nakamatsu "A quantum bi-directional self-organizing neural network (QBDSONN) architecture for binary object extraction from a noisy perspective," Applied Soft Computing, vol.46, pp. 731-752, 2016, DOI: https://doi.org/10.1016/j. asoc.2015.12.040.

[38] D. Konar, S. Bhattacharya, U. Chakraborty, T. K.Gandhi, B. K. Panigrahi, "A quantum parallel bi-directional self-organizing neural network (QPBDSONN) architecture for extraction of pure color objects from noisy background," Proc. IEEE International Conference on Advances in Computing, Communications and Informatics (ICACCI), 2016, pp. 1912 1918, 2016, DOI: 10.1109/ICACCI.2016.7732330.

[39] D. Konar, S. Bhattacharyya and B. K. Panigrahi, "QIBDS Net: A Quantum-Inspired Bi-Directional Self-supervised Neural Network Architecture for Automatic Brain MR Image Segmentation," Proc. 8th International Conference on Pattern Recognition and Machine Intelligence (PReMI 2019), vol. 11942, pp. 87-95, 2019, DOI:https://doi.org/10.1007/ 978-3-030-34872-4_64.

[40] M. A. Nielson and I. L. Chung, Quantum computation and quantum information, Cambridge University press, 2002.

[41] T. G. Kolda, B. W. Bader, "Tensor Decompositions and Applications," SIAM Review, vol. 51, no. 3, pp. 455--500, 2009, DOI: https://doi.org/ 10.1137/07070111X

[42] S. Bhattacharyya, P. Dutta and U. Maulik, "Multilevel image segmentation with adaptive image context based thresholding," Applied Soft Computing, vol. 11, no.1, pp. 946-962, 2011, DOI: https://doi.org/10. 1016/j.asoc.2010.01.015.

[43] N. Otsu, "A threshold selection method from gray level histograms," IEEE Transactions on Systems, Man, and Cybernetics, vol. 9, no. 1, pp. 62-66, 1979, DOI: 10.1109/TSMC.1979.4310076.

[44] H. P. Ng, S. H. Ong, K. W. C. Foong, P. S. Goh, and W. L. Nowinski, "Medical Image Segmentation Using K-Means Clustering and Improved Watershed Algorithm," 2006 IEEE Southwest Symposium on Image Analysis and Interpretation, 2006, DOI: 10.1109/SSIAI.2006.1633722.

[45] B. H. Menze et al., "The multimodal brain tumor image segmentation benchmark (BRATS)," IEEE Transactions on Medical Imaging, vol. 34, no. 10, pp. 1993--2024, 2015, DOI: 10.1109/TMI.2014.2377694.

[46] P. Bilic et al., "The Liver Tumor Segmentation Benchmark (LiTS)," 2019, arXiv: 1901.04056

[47] M. H. Gail and S. B. Green, "Critical values for the one-sided twosample Kolmogorov-Smirnov statistic," J. Am. Stat. Assoc., vol. 71, pp. 757-760, 1976.

[48] H. Kim, J. Kang, W. Park, S. Ko, Y. Cho, D. Yu, Y. Song, and J. Choi, "Convergence Analysis of Optimization Algorithms," 2017, arXiv:1707. 01647. 